\title{
Ultradian rhythms in the perception of the spiral aftereffect
}

\author{
PERETZ LAVIE, C. MICHAEL LEVY, and FREDERICK L. COOLIDGE \\ University of Florida, Gainesville, Florida 32611
}

\begin{abstract}
Young adults were tested on the spiral aftereffect (SAE) for 8-h periods during the day and night. Using time series analyses, prominent cyclicity components in the perception of the illusion were revealed, with periodicities of the SAE the same order of magnitude as the REM-nonREM cycle. It appeared that the duration of the SAE could be used as a sensitive behavioral index of the basic rest-activity cycle.
\end{abstract}

In the decade since Kleitman (1963) originally hypothesized that there was an underlying biological rhythm of which the REM-nonREM cycle is the nightly manifestation, only shards of data have materialized. Support for this basic rest-activity cycle (BRAC) model, however, has been emerging recently from diverse methodologies. Human adults (Friedman \& Fisher, 1967; Oswald, Merrington, \& Lewis, 1970) and cats (Sterman, Lucas, \& MacDonald, 1972) have been shown to have a diurnal rhythm of "oral intake" with the same periodicity as the REM-nonREM cycle, and a rest-activity cycle was demonstrated in human fetuses with the same periodicity as the REM-nonREM cycle of the newborn infant (Sterman, 1972). A different approach to establish the independence of REM and nonREM sleep per se from sleep and wakefulness involved the demonstration of carry-over effects from the two stages of sleep into the waking state. Thus, when awakened from REM sleep, subjects produced more bizarre and vivid responses to the thematic apperception test than when awakened from nonREM sleep (Fiss, Klein, \& Bokert, 1966), and when awakened from the beginning of REM sleep, displayed longer durations of the spiral aftereffect (SAE) than after arousal from the beginning of nonREM sleep (Stage 4) (Lavie \& Giora, 1973). Similar differential durations of the SAE illusion, persisting for periods in excess of $15 \mathrm{~min}$ were found upon awakening from the beginning of REM sleep and 5 min after the end of REM sleep (Lavie, 1974).

These results suggested that the possible existence of "REM-like" periods during the waking state would be associated with longer durations of the SAE as compared to corresponding "nonREM" periods. Thus, it was anticipated that when subjects were continuously tested during the waking state, the duration of the illusion would vary in a cyclical manner across the $24 \mathrm{~h}$ with a

The authors acknowledge their appreciation to the Social Science Institute of the University of Florida for providing financial support for the project, to Professor W. B. Webb for his critical advice, and to Ms. C. Edge and Mr. H. J. Darling for their technical assistance. Dr. Peretz Lavie is presently at the University of California at San Diego. Address reprint requests to Dr. P. Lavie, VA Hospital, 3350 La Jolla Village Drive, San Diego, California 92161. periodicity approximating that of the REM-nonREM cycle. In order to evaluate this hypothesis, subjects were tested on the SAE continuously in 8-h sessions extending over both day and night periods.

\section{METHOD}

\section{Subjects}

The subjects were six females and two males, ages 19-24 years, who were completely naive with respect to the experimental hypotheses. Service in the experiment was voluntary and without payment.

\section{Procedure}

In the first part of the experiment, subjects slept for three nonconsecutive nights in the laboratory. Upon their arrival at 11:00 p.m. each night, they were fitted with electrodes according to the International 10-20 system and were subsequently given an explanation of the SAE illusion and permitted $15 \mathrm{~min}$ practice with it. The SAE apparatus was a $7-\mathrm{cm}$ Archimedes spiral centered and held on the shaft of a rotor by a $1-\mathrm{cm}$ knob. The subjects were instructed to fixate the central knob as the spiral rotated and to continue this fixation after it ceased spinning. When the apparent expansion of the spiral (i.e., the illusion) terminated, they were to press a microswitch which stopped a timer located in an adjacent room. They were carefully instructed to use the same criterion for the persistence of the illusion on every trial. After this practice period, the house lights were extinguished, and the subjects were permitted to sleep from 12:00 p.m. to 8:00 a.m. during the first two nights. Following the third night, they were awakened at approximately 7:50 a.m. and tested on the SAE twice each $5 \mathrm{~min}$ from 8:00 a.m. to 4:00 p.m. No attempt was made to limit the subjects' activities other than confining them to the general laboratory area during the test phase, where they spent most of the intertrial time reading or listening to music. Meals and snacks were available to the subjects ad lib. Because the demands upon the subjects were heavy, they were permitied to select, during the course of the practice periods, one of three durations which would be used exclusively during the 8-h testing period. Two subjects were given $5 \mathrm{sec}$ of rotation, five subjects were given $10 \mathrm{sec}$ of rotation, and one subject was given $15 \mathrm{sec}$ of rotation. In all tests, the rotation of the spiral was in counterclockwise direction at $100 \mathrm{rpm}$. As anticipated, no cyclicity differences were observed as a consequence of length of rotation.

In the second part of the experiment, the original subjects returned at least 7 days after their previous service and were tested on the SAE in the same manner as before, but from the period between 12:00 p.m. and 8:00 a.m. The subjects were 


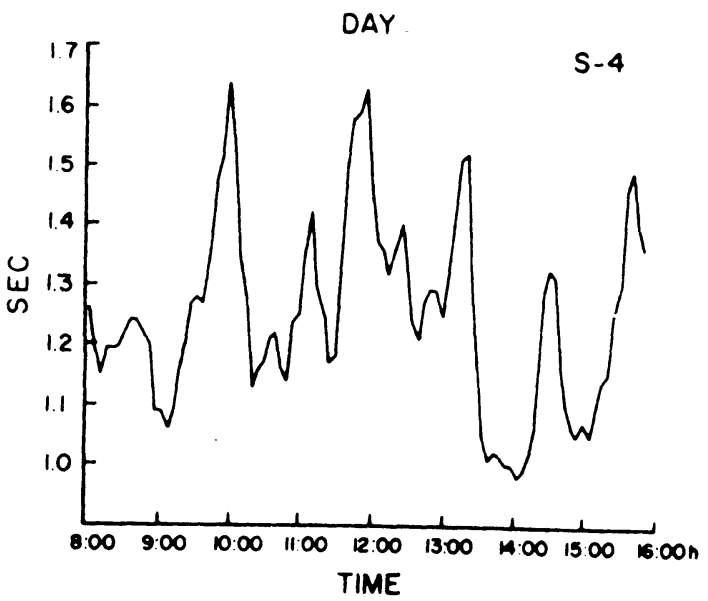

Figure 1. Four-point moving average spiral aftereffect cycles for Subject 4 for daytime period.

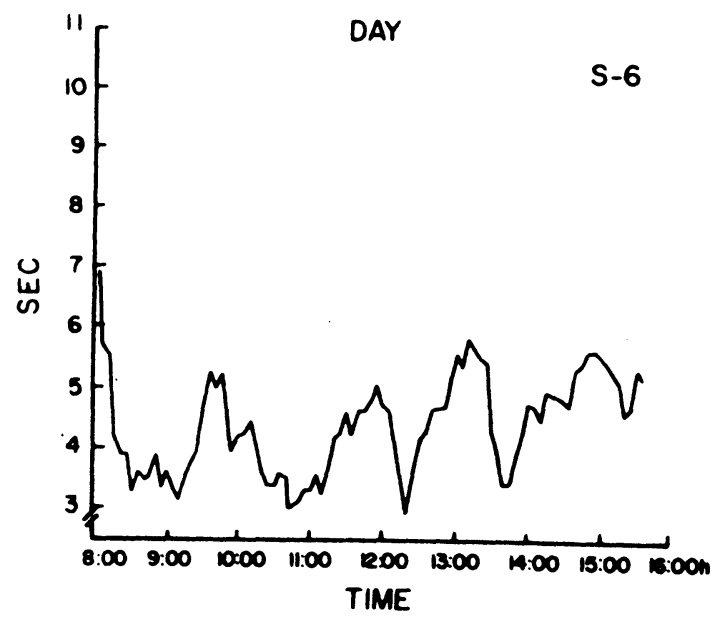

Figure 2. Four-point moving average spiral aftereffect cycles for Subject 6 for daytime period.

instructed to try to have a "normal" day prior to this session and not to take a nap prior to their appearance at the laboratory. One subject complained of the heavy demands and was excused after $2 \mathrm{~h}$ of participation in the second experiment. His data were excluded from the analysis.

\section{RESULTS}

Each subject provided two measures of the duration of the illusion every $5 \mathrm{~min}$. After obtaining the mean of each of these pairs of trials, one time series was constructed for each subject for each of the 8-h test sessions. Figures 1 and 2 display two of these time series. For presentation purposes, the time series were smoothed by a moving average process with $\mathrm{N}=4$. Visual observation of the time series revealed that Subject 4 had five peaks during the day time time series; the first appeared at 10:00 a.m. and subsequent peaks appeared after $115,80,75$, and $70 \mathrm{~min}$. The mean interpeak interval was $85 \mathrm{~min}$. Subject 6 also displayed five clear peaks, beginning his cycle from a high phase and reaching subsequent peaks after 100, 140,75, and $95 \mathrm{~min}$; his average interpeak interval was $102.5 \mathrm{~min}$. The two subjects manifested reliably different durations of the illusion: the mean durations for Subject 4 and Subject 6 were $1.25 \mathrm{sec}$ and $4.42 \mathrm{sec}$, respectively.

To determine the primary periodicities of the time series, the spectral analysis technique was employed. First, the time series were detrended to eliminate the linear regressions which were found to be significant for most of the time series; then, the autocorrelation coefficients were calculated for 20 lags. Subsequently, the power spectral estimates were calculated for 20 spectral frequencies ranging from 10 to $200 \mathrm{~min}$, using the Hanning window.

Observing the spectral density functions revealed that four out of the seven day-time series had spectral peaks at the frequencies $100 \mathrm{~min} /$ cycle (3) and $80 \mathrm{~min} /$ cycle (1). While three of these peaks were the highest spectral peaks, one (100 min/cycle) was a secondary peak, additional to a peak of the same magnitude at $33 \mathrm{~min} /$ cycle. Of the remaining three times series, two revealed the maximum power for the 0 frequency, and one had a broad spectral peak over the frequencies 100 and $200 \mathrm{~min} / \mathrm{cycle}$ and the 0 frequency. Similarly, four out of the seven night-time series revealed spectral peaks at the frequency $100 \mathrm{~min} /$ cycle; of these, three were the maximum spectral peaks and one was approximately equal to an additional peak at the frequency $10 \mathrm{~min} / \mathrm{cycle}$. One night-time series had the maximum power for the 0 frequency, while the remaining two night time series revealed broad spectral peaks over several frequencies including the $100 \mathrm{~min} /$ cycle frequency.

In summary, 6 out of the 14 time series had the maximum spectral peaks at the frequencies $100 \mathrm{~min} / \mathrm{cycle}$ (5), and $80 \mathrm{~min} / \mathrm{cycle}$ (1), and, two additional times series revealed secondary $100 \mathrm{~min} / \mathrm{cycle}$ peaks. Thus, $57 \%$ of the time series demonstrated primary or secondary spectral peaks for periodicities repeatedly reported for the REM-nonREM cycle during sleep. Regarding the day-night differences, all of the subjects demonstrated one ultradian peak for only one of the test periods, excluding two subjects, one of whom demonstrated the maximum power for the 0 frequency for both test periods, and the other subject who demonstrated the same primary peaks $(100 \mathrm{~min} /$ cycle $)$ for both periods. Figure 3 presents the power density functions of the two times series displayed in Figures 1 and 2. Both functions revealed prominent spectral peaks at the frequency $100 \mathrm{~min} / \mathrm{cycle}$.

Two approaches were utilized to determine whether the distribution of power over the 20 spectral frequencies and 14 time series represent a random 


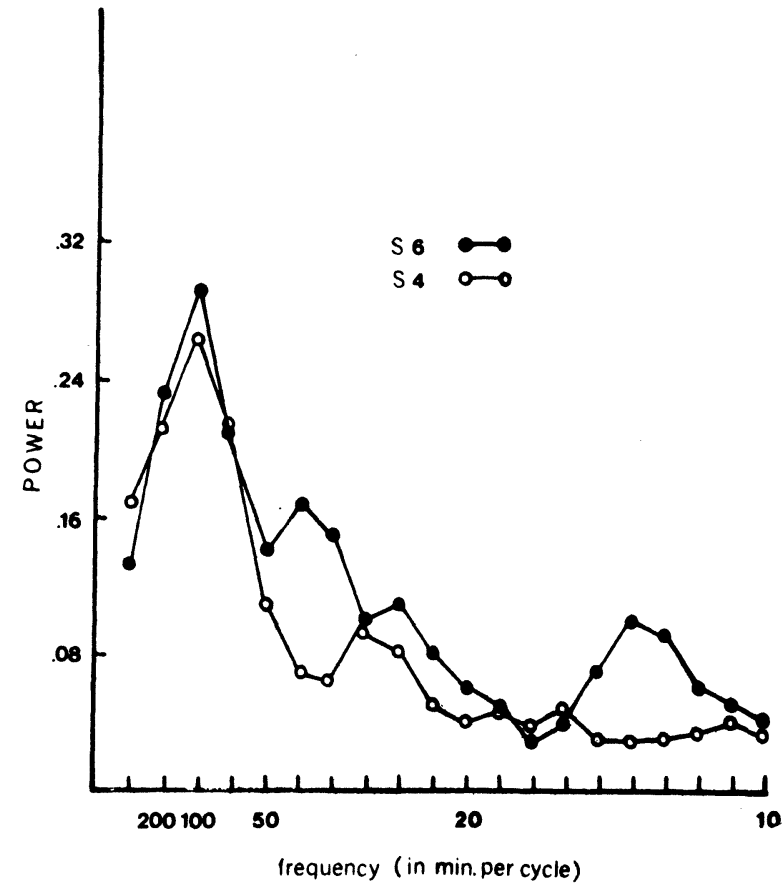

Figure 3. Power density functions of the spiral aftereffect cycles presented in Figures 1 and 2.

distribution. First, the normalized power for the mode spectral peak frequency $(100 \mathrm{~min} / \mathrm{cycle})$ was compared to the average power in the rest of the 19 spectral frequencies. All the subjects had a higher power for this frequency for both the day and night test periods. The mean power for the $100 \mathrm{~min} /$ cycle frequency exceeds the average power in the rest of the frequencies by $91.5 \%$ and $96.36 \%$ for the day and night-time series, respectively. These differences were found to be significant for both day $(\mathrm{t}=3.02, \mathrm{df}=6, \mathrm{p}<.025)$ and night $(\mathrm{t}=5.0, \mathrm{df}=6, \mathrm{p}<.005)$ comparisons. Second, since 5 out of the 14 times series had the highest spectral peak at one out of the 20 spectral frequencies (100 $\mathrm{min} /$ cycle $)$, it was possible to utilize the binomial theorem to assess the probability of such an event being random (Kripke, 1972). This probability came to be less than .00006 .

\section{DISCUSSION}

The results provide strong evidence for the existence of ultradian rhythms in the perception of the spiral aftereffect with periodicities similar to those of the REM-nonREM cycle during both night and day periods. Six of the 14 times series had a primary ultradian peak, while two more had a secondary ultradian peak. Moreover, the periodicity in perception was found to be independent of the absolute duration of the illusion, as seen in Figures 1 and 2. If one tends to accept earlier speculations (Eysenck, 1957) that changes in the duration of the spiral aftereffect reflect changes in cortical arousability level, the results strongly support Kleitman's original hypothesis (1963) that the REM-nonREM cycle is a part of a basic rest-activity cycle that can be detected during the waking state as periodic fluctuations in the level of alertness.

Several reasons might account for the fact that subjects did not demonstrate clear periodicities on both test periods. First, subjects' activity in the present study was not restricted in any way, as opposed to other experimental designs (e.g., Kripke, 1972) investigating the ultradian periodicity. Subjects could read, listen to music, or interact with the experimenter. Thus, variable levels of subjects' activity could mask the ultradian periodicity for some subjects. On the other hand, the heavy experimental demands induced fatigue and boredom, which resulted in a continuous reduction in the level of the illusion, and hence, it might be that in some cases, the ultradian rhythmicity was masked by the strong linear trends. Finally, the fact that two subjects demonstrated consistent patterns of response over the night and day periods might suggest that there are individual differences in the vulnerability of the waking ultradian rhythm, as was recently observed by Hiatt and Kripke (paper submitted to press), with respect to ultradian rhythms in gastric contractions of fasting subjects;

In summary, the fact that six out of the seven subjects demonstrated primary or secondary ultradian peaks, in spite of the heavy experimental demands, and the lack of an isolation paradigm strongly commends the spiral aftereffect as an efficient tool in studying the waking counterpart of the REM-nonREM cycle.

\section{REFERENCES}

Eysenck, H. J. Drugs and personality: I. Theory and methodology. Journal of Science, 1957, 103, 119-131.

Fiss, H., Klein, G. S., \& Bokert, E. Waking fantasies following interruption of two types of sleep. Archives of General Psychiatry, 1966, 19, 543-551.

Friedman, S., \& Fisher, C. On the presence of a rhythmic diurnal and oral instinctual drive cycle in man. Journal of the American Psychoanalytic Association, 1967, 15, 317-343.

kleitman, N. Sleep and wakefulness. Chicago: University of Chicago Press, 1963.

Kripke, D. F. An ultradian biologic rhythm associated with perceptual deprivation and REM sleep. Psychosomatic Medicine, 1972, 3, 221-234.

Lavie, P. Differential effects of REM and nonREM awakenings on the spiral aftereffect. Physiological Psychology, 1974, 2, 107.

Lavie, "P., \& Giora, Z. Spiral aftereffect durations following awakening from REM sleep and nonREM sleep. Perception \& Psychophysics, 1973, 1, 19-20.

Oswald, I., Merrington, J., \& Lewis, H. Cyclical "on demand" oral intake by adults. Nature, 1970, 225, 959-960.

Sterman, M. B. The basic rest-activity cycle and sleep: Developmental considerations in man and cats. In C. C. Clemente, D. Purpora, F. Mayer (Eds.), Sleep and the maturing nervous system. New York: Academic Press, 1972.

Sterman, M. B., Lucas, E. A., \& MacDonald, L. R. Periodicity within sleep and operant performance in the cat. Brain Research, 1972, 38, 327-341.

(Received for publication March 26, 1974; revision received October $30,1974$. 